\title{
e-Phaïstos
}

e-Phaïstos

Revue d'histoire des techniques / Journal of the history

of technology

I-2 | 2012

Les sources de l'histoire des techniques

\section{Les évolutions de la notion d'entreprise}

Entretien avec Serge Benoit

Serge Benoit, Guy-Grégoire Awono-Zinga et Benjamin Ravier-Mazzocco

\section{Q OpenEdition}

1 Journals

Édition électronique

URL : http://journals.openedition.org/ephaistos/440

DOI : 10.4000/ephaistos.440

ISSN : 2552-0741

Éditeur

IHMC - Institut d'histoire moderne et contemporaine (UMR 8066)

Édition imprimée

Date de publication : 1 décembre 2012

Pagination : 79-84

ISSN : 2262-7340

\section{Référence électronique}

Serge Benoit, Guy-Grégoire Awono-Zinga et Benjamin Ravier-Mazzocco, « Les évolutions de la notion d'entreprise », e-Phaïstos [En ligne], I-2 | 2012, mis en ligne le 12 février 2016, consulté le 14 septembre 2020. URL : http://journals.openedition.org/ephaistos/440 


\title{
Entretien avec Serge Benoit
}

\section{Les évolutions de la notion d'entreprise}

\author{
Par : \\ Guy-Grégoire Awono-Zinga \\ et \\ Benjamin Ravier-Mazzocco
}

\section{Cursus académique}

1970-1975 : Ancien élève de l'Ecole Normale Supérieure (rue d'Ulm).

1970 : Licencié de Lettres Classiques, Faculté des Lettres et Sciences humaines de

Paris.

1971 : Licencié d'histoire, Université de Paris-1

1972 : Maîtrise d'Histoire, Université de Paris-1

1974 : Reçu à l'Agrégation d'Histoire,

1976-1983 : Professeur agrégé d'Histoire-Géographie au Lycée d'Etat d'Evreux (Eure)

1983-1996 : Chargé de recherches au CNRS, en détachement, 1983-1996 (Centre de Recherches Historiques, EHESS-CNRS)

1996-2008 : Professeur agrégé d'histoire à l'Université d'Evry Val d'Essonne.

2006 : Reçu Docteur en histoire moderne et contemporaine de l'Université d'Evry Val d'Essonne, 2006, avec les félicitations à l'unanimité du jury. Thèse intitulée : «La modernité de la tradition : Le rôle des énergies renouvelables classiques - l'eau et le bois -, dans la voie française de l'industrialisation, c. 1780 - c. 1880,1440 p., sous la direction du Professeur Jean-Louis LOUBET. depuis 2008 : Maître de conférences en histoire mo- derne et contemporaine à l'Université d'Evry depuis 2008.

Responsabilités associatives dans le domaine du patrimoine industriel

1978-1998 : Secrétaire de l'Association pour la sauvegarde et l'animation des Forges de Buffon

1984-1994 : Secrétaire général du CILAC

\section{Bibliographie sélective}

co-éditeur, avec Jean GAYON et al., de : Buffon 88, Actes du Colloque International Paris-Montbard-Dijon, Paris, Librairie Vrin, 1992, 770 p.

Co-éditeur avec Jean-François BELHOSTE, Serge CHASSAGNE et Philippe MIOCHE de : Autour de l'industrie, histoire et patrimoine. Mélanges offerts à Denis Woronoff, Paris, Comité pour l'histoire économique et financière de la France, 2004, XVI-640 p.

Co-éditeur avec Gérard EMPTOZ et Denis WORONOFF de : Encourager l'innovation en France et en Europe. Autour du bicentenaire de la Société d'encouragement pour l'industrie nationale, Paris, Editions du CTHS, 2006, 379 p. 
e-Phaïstos : À quand feriez-vous remonter la notion d'entreprise?

Serge Benoit : À mon sens, les notions d'entreprise et d'entrepreneur sont plus anciennes que les définitions qu'en donnent les fondateurs de l'économie politique du XVI ${ }^{\mathrm{e}}$ au XVIII ${ }^{\mathrm{e}}$ siècle. Je vois apparaitre la notion d'entreprise dès le Moyen Âge dans le sens de contrat de travail, de tâche confiée à quelqu'un, comme le montre très bien le juriste Philippe Didier. Cela signifie que l'entreprise est une réalité de nature temporaire, strictement liée à la réalisation de la tâche commandée. Les documents médiévaux nous offrent de très nombreux exemples où un pouvoir public confie la construction d'un château, ou une institution ecclésiastique la construction d'une église, à un maître-bâtisseur par l'intermédiaire d'un « contrat d'entreprise », qui prévoit les conditions financières (coût, rétribution) à la réalisation de cette entreprise, par nature temporaire. La notion d'entreprise, très ancienne, garde d'ailleurs longtemps un aspect économique positif, l'aspect subversif de l'entrepreneur ne se développant que plus tardivement comme le note très bien Hélène Vérin.

Notez bien que cette notion d'entreprise temporaire, de nature contractuelle, est quelque chose de fort, et qui n'a pas disparu, puisqu'encore aujourd'hui, on parle d'entreprise générale pour une société qui va se voir précisément confier de grands travaux publics comme, par exemple, la construction d'une autoroute. Cette entreprise générale est par ailleurs une entreprise au sens moderne du mot : une institution permanente qui assure des fonctions économiques, mais qui retrouve aussi cette idée ancienne d'une tâche temporaire confiée à quelqu'un. Ainsi, des sociétés aussi célèbres que Bouygues ou Vinci ayant des fonctions d'entreprise générale, doivent assumer l'héritage lointain de cette notion médiévale.

$\boldsymbol{e}$-Phaïstos : Derrière ces contrats passés pour la construction de bâtiments, est-ce que la personne à qui on confie la tâche coordonne déjà une sorte d'organisation?
S. B. : C'est une personne privée, en général individuelle, qui ensuite va se charger elle-même de recruter les salariés dont elle aura besoin pour faire la construction. Pour autant, il n'y a pas cette idée de permanence dans l'entreprise qu'on va voir se développer dès les XVIII $^{\mathrm{e}}$ et $\mathrm{XIX}^{\mathrm{e}}$ siècles. En effet, l'entrepreneur est d'abord quelqu'un qui est capable de faire des constructions, et qui a un savoir-faire solide. Il est d'ailleurs possible de voir, y compris dans les activités plus techniques comme par l'exemple la métallurgie, des artisans spécialisés prendre un contrat d'entreprise : par exemple, lorsqu'il s'agit de fournir des éléments en fer pour un château, c'est bien le forgeron qui va passer ce contrat.

$\boldsymbol{e}$-Phaïstos : Peut-on appeler ces personnes des entrepreneurs?

S. B. : Entrepreneurs au sens de l'époque, oui, puisque ce sont eux qui réalisent un contrat. Le mot entrepreneur a ici une dimension purement juridique.

Sur ce point, apparaissent à la fin de la période moderne des ambigüités entre les formulations françaises et les formulations des autres langues étrangères. Par exemple, lors du développement de la proto-industrialisation à l'époque moderne, on trouve la notion allemande d'entreprise sous ce qu'on appelle le Verlagssystem. Le Verlagssystem va organiser la protoindustrialisation, entendue comme système de la manufacture dispersée autour d'un Verlager, qui va distribuer du travail aux paysans. Ce mot, employé dans la langue allemande à partir du XVI ${ }^{\mathrm{e}}$ siècle si ce n'est auparavant, correspond à la notion d'entreprise en français. Pour autant, en France, pour désigner la même activité, on va plutôt employer une autre notion : celle de marchand-fabricant. Ainsi le mot qui correspond à entreprise en allemand a désigné assez tôt une réalité, qui existait aussi en France mais qui n'y prenait pas le même sens. Le sens allemand de l'entreprise insiste donc davantage sur la sous-traitance et la coordination des travaux, que ne le font les notions française d'entrepreneur, ni celles de marchand-fabricant. 
e-Phaïstos : Dans un acte notarié, on demandait de réaliser une entreprise de construction à un maçon par exemple, mais quand va-t-on le demander à un entrepreneur? À quel moment le nom se raccroche-t-il à une personne et qu'est ce que cela change-t-il dans la façon de percevoir l'entrepreneur?

S. B. : L'entrepreneur est celui qui, par définition, «prend » le travail : il y a " prise » dans entreprise, c'est donc quelqu'un qui accepte de faire un travail demandé. D'où la connotation de dépendance, de subordination, que comporte le terme allemand d'Unternehmer ou encore le terme anglais d'undertaker. Nous avons vu que, dans d'autres terminologies, on va insister davantage sur la fonction de sous-traitance, l'entrepreneur étant alors celui qui va passer le contrat, le proposer. Dans la terminologie française, depuis le Moyen Âge, l'entrepreneur se trouve quant à lui dans une situation subordonnée par rapport au contrat d'entreprise proposé par le commanditaire. Je pense que la perception de l'entrepreneur bascule au moment où la prise d'un travail, sa mise en œuvre, n'est plus synonyme de subordination. En effet, de nos jours, l'entrepreneur est avant tout celui qui dirige son entreprise. Ainsi, bien qu'il passe contrat avec un client, on ne peut plus dire, à l'instar de notre maçon médiéval, qu'il est dans une position subordonnée.

e-Phaïstos : À quel moment bascule-t-on de cette notion de contrat pris à cette notion plus moderne d'entrepreneur, se rapprochant de celle de capitaine d'industrie?

S. B. : À mon avis, ce basculement s'opère au XVIII ${ }^{\mathrm{e}}$ siècle. On en a la démonstration dans l'Encyclopédie méthodique avec ce qu'écrit Roland de la Platière, le futur ministre girondin qui était alors inspecteur des manufactures, qui définit l'entrepreneur comme " l'homme qui est à la tête d'un établissement en grand où l'on emploie diverses sortes de matières, ou d'un établissement où l'on modifie très diversement la même matière ». C'est la fonction de direction qui tend désor- mais à l'emporter dans la définition du rôle de l'entrepreneur. Il est alors très clair que vers 1780 , et probablement avant, l'entrepreneur devient celui qui assure la coordination de fonctions techniques complexes dans un établissement, depuis la manufacture des Gobelins au XVII ${ }^{e}$ siècle, jusqu'à l'indiennage de la fin du XVIII ${ }^{e}$ siècle illustré par Christophe-Philippe Oberkampf. La notion d'entreprise n'est ici plus reliée à cette idée de contrat, mais à celle de direction d'opérations industrielles complexes et où une coordination du travail doit être assurée. C'est à partir de là qu'on peut faire commencer la mutation.

$\boldsymbol{e}$-Phaïstos : Est-ce que derrière cette idée de direction, il y a la nécessité, pour un entrepreneur, de garder un minimum de savoir-faire?

S. B. : Rappelons qu'au XVIII ${ }^{\mathrm{e}}$ siècle, le savoir-faire reste encore fondamentalement entre les mains des ouvriers. Dans une manufacture comme celle de Sedan, il y a bien un entrepreneur, c'est-à-dire quelqu'un qui va diriger l'entreprise, mais le savoir-faire technique reste encore fondamentalement entre les mains des différentes catégories ouvrières dont chacune détient une qualification spécifique très identifiée. L'entrepreneur est celui qui dirige effectivement l'entreprise en question, et qui a, à ce titre, une fonction de coordination des ouvriers. Cela dit, il n'intervient pas dans le processus de production, et ne possède pas obligatoirement de savoir-faire particulier. Il fait évidemment en sorte qu'il y ait une cohérence entre toutes les étapes de la fabrication, et surtout, il maintient l'ordre, faisant ici jouer pleinement son rôle social de direction, surtout quand des ouvriers mécontents se mettent en grève. Le regretté Gérard Gayot avait insisté sur le caractère déjà conflictuel de l'entreprise à ce niveau là.

Fondamentalement, l'entrepreneur de l'époque moderne est un commerçant, c'est un capitaliste commercial qui n'a pas de compétences techniques spéciales, qui doit effectivement tenir compte du savoir-faire des ouvriers et qui est obligé de les payer correctement, s'il veut les conserver face à la concurrence. Cette dimen- 
sion commerciale continue d'apparaître jusqu'assez tard dans le XIX ${ }^{\mathrm{e}}$ siècle, même si la notion d'entreprise insiste davantage sur le mode de gestion. Ainsi, l'État va faire gérer les manufactures qu'il possède, et notamment les manufactures militaires, par des entrepreneurs privés. C'est ce qu'on appelle le " régime de l'entreprise » : un régime dans lequel l'État demeure propriétaire, tout en en confiant la gestion à un entrepreneur local. C'est par exemple le cas la manufacture d'armes de Châtellerault (Vienne). Première grande manufacture d'armes blanches hydraulique, construite sous la Restauration, loin de la frontière allemande, elle sera confiée en location aux dirigeants des établissements de coutellerie de la région, comme Proa, qui employaient déjà plusieurs centaines d'ouvriers. On en revient à l'alternative de gestion qui avait déjà été posée à la fin de l'Ancien Régime pour les manufactures militaires, entre régie directe par l'État et location à des entrepreneurs privés désignés comme tels. Il y avait donc un certain savoir-faire de la gestion d'un personnel qualifié et parfois revendicatif.

Prenons ici l'exemple d'Edison, celui-ci n'est probablement pas le propriétaire des mille brevets ou davantage qu'il a fait déposer en son nom propre par son bureau d'études. En revanche, il a eu suffisamment de génie pour assurer la coordination au sein d'un bureau d'études particulièrement diversifié réunissant entre autres métallurgistes et électriciens. C'était donc un bon entrepreneur en ce qu'il était capable de gérer cette grande variété de compétences, avec cette idée persistante qu'il ne peut pas y avoir d'invention chez Edison qui ne débouche pas sur quelque application rentable. C'est ce qui a fait sa grande force dans l'affaire de l'éclairage électrique de la bourse de Wall Street. C'est aussi ce qui va le pousser à abandonner l'industrie cinématographique, alors trop peu rentable.

$\boldsymbol{e}$-Phaïstos : Un entrepreneur pouvait-il être aussi propriétaire de l'entreprise dirigée ?

S. B. : Oui, bien sûr, mais je souligne ici le fait que la notion d'entreprise insiste sur la gestion. Cette notion d'entrepreneur comme gestionnaire est d'une grande importance idéologique. En effet, un certain nombre d'économistes, de théoriciens et d'idéologues du XIX siècle insistent beaucoup sur cette notion de direction. Avec Jean-Baptiste Say, par exemple, tout le discours tenu à partir de son traité de 1817 insiste précisément sur le fait que l'entrepreneur c'est le chef d'entreprise ; c'est-à-dire celui qui dirige, celui qui coordonne les différentes fonctions. Cela n'exclut pas pour autant qu'il soit propriétaire, mais cette insistance caractéristique est aussi l'une de ses différences avec Marx. Pour Marx, le capitaliste est forcément celui qui possède le capital alors que pour Say, la dimension de gestion et de direction prime dans le système économique. Au XIX ${ }^{\mathrm{e}}$ siècle le capitaliste est celui qui apporte son argent et l'entrepreneur c'est celui qui dirige l'entreprise. Le bailleur de fonds constitue l'image du capitaliste dans la vision de l'époque de Louis-Philippe et de Balzac. L'économiste, un peu oublié aujourd'hui, J.-G. Courcelle-Seneuil, auteur en1857 d'un Traité théorique et pratique des entreprises industrielles, commerciales et agricoles, se situe dans la même veine que J.-B. Say, tout en se plaçant dans une perspective anthropologique lorsqu'il définit la notion d'entreprise comme « toute application de l'activité humaine qui consiste à combiner l'emploi de forces diverses pour atteindre un but déterminé ».

$\boldsymbol{e}$-Phaïstos : Est-ce que les compétences de l'entrepreneur devaient-elles être forcément compatibles avec les activités de l'entreprise qu'il dirigeait ?

S. B. : En effet, même s'il est vrai que l'entrepreneur est d'abord un gestionnaire, il est indispensable qu'il soit capable de comprendre ce que les techniciens font. Il lui faut donc un minimum de connaissances. Il est certain par exemple l'entrepreneur au sens de Roland de la Platière doit pouvoir comprendre ce que ces ouvriers sont capables d'exécuter et même de le critiquer si nécessaire, alors même qu'il n'a pas l'expérience pratique de la matière, le savoir-faire physique lui restant inconnu. L'entrepreneur dispose ainsi d'une certaine autorité, qui lui permet de coordonner le travail des différents ouvriers.

Prenons un autre cas du rôle de l'entrepreneur au 